\title{
"Bell's Palsy: An Unusual Complication Secondary To Odontogenic Space Infection A Case Report"
}

\author{
Dr. Samir,D. Khaire ${ }^{1}$, Dr. Shameeka S. Khaire ${ }^{2}$ \\ ${ }^{1}$ Maxillofacial \& Oral Surgeon, Assistant Professor, Department Of Dentistry, Byramjee Jeejeebhoy \\ Government Medical College \& Sassoon General Hospitals’ Pune \\ ${ }^{2}$ (Thopte), Assistant Professor In Oral Medicine, Diagnosis \& Radiology, Bharati Vidyapeeth Deemed \\ University Dental College \& Hospital, Pune.
}

\section{Introduction}

In 1550 Gabriel Fallopius identified the anatomical course of the facial nerve within the temporal bone and recognized the chorda tympani nerve as a branch of the facial nerve. In 1829 Charles Bell properly attributed sensory innervation to the $\mathrm{V}^{\text {th }}$ cranial nerve and motor innervation to the $\mathrm{VII}^{\text {th }}$ cranial nerve. In 1821 , Charles Bell demonstrated that the Vth and the VIIth nerves separately distributed their fibres predominantly to the facial skin and muscles respectively. ${ }^{1}$

\section{Case Report}

A male patient, 31 years old, residing at Mankhurd, driver by occupation, had reported with the complaint of swelling on left side of face since 8 to 10 days.Patient said that he was apparently alright 10 days back. He gave H/O sharp, shooting pain with mandibular left posterior teeth. He gave H/O hot foamentation following which there was swelling, which gradually increased in size. He gave no $\mathrm{H} / \mathrm{O}$ any difficulty in breathing or swallowing. He gave H/O fever 2 days following the swelling on the face.PAST MEDICAL HISTORY: Patient had suffered from Koch's disease 2 years back. Anti-Koch's Treatment was taken for 14 months. He gave $\mathrm{H} / \mathrm{Ogain}$ in weight, increased appetite following treatment.

Past Dental History: He gave H/O dental extraction done under local anaesthesia few years back as the teeth were carious. No $\mathrm{H} / \mathrm{O}$ any complications intraoperatively as well as postoperatively.

Family History: Nothing significant

Personal History: Patient said he had used a toothpick for removing the impacted food from the last tooth of the lower jaw on left side. H/O trauma to the gums during removal of the impacted food.

Examination: Patient was conscious, co-operative and well oriented in time, place and person.General condition was moderate, febrile Pulse rate 100 beats/minute Blood pressure 110/70 mm of $\mathrm{Hg}$ Swelling on left side of face. Swelling was soft, fluctuant in the region of left angle of mandible.

Skin overlying the swelling was warm. Patient was unable to open mouth. Mandibular left third molar was partially visible in the oral cavity. Intraoral pus discharge was evident from mandibular left third molar region from buccal as well as lingual side.

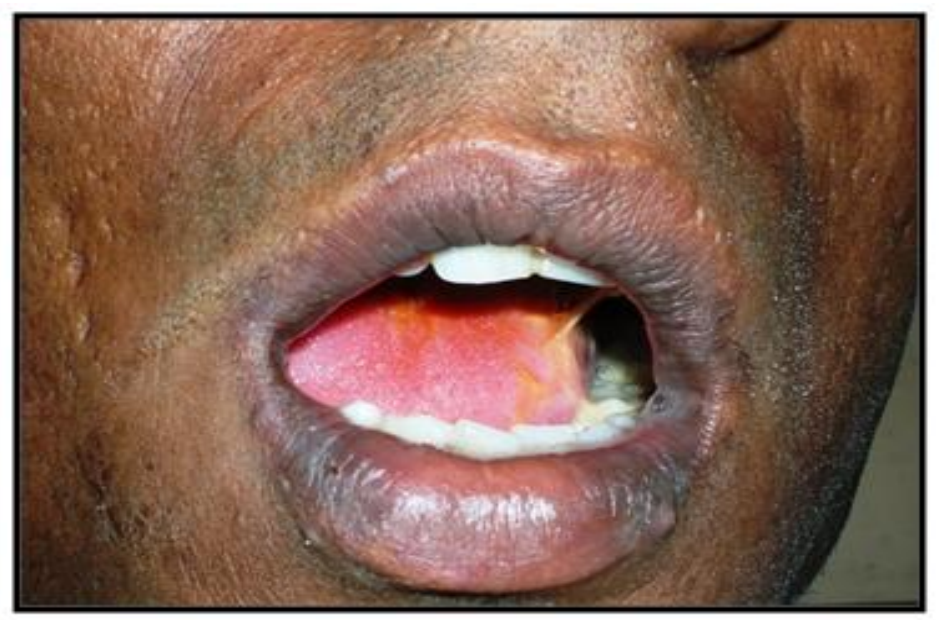

Figure showing copious amount of pus discharge seen intraorally 
Facial nerve paralysis was evident on left side. (However, patient reported that before the swelling appeared, he was able to close his eyes, blow his mouth and produce wrinkles on the forehead.)
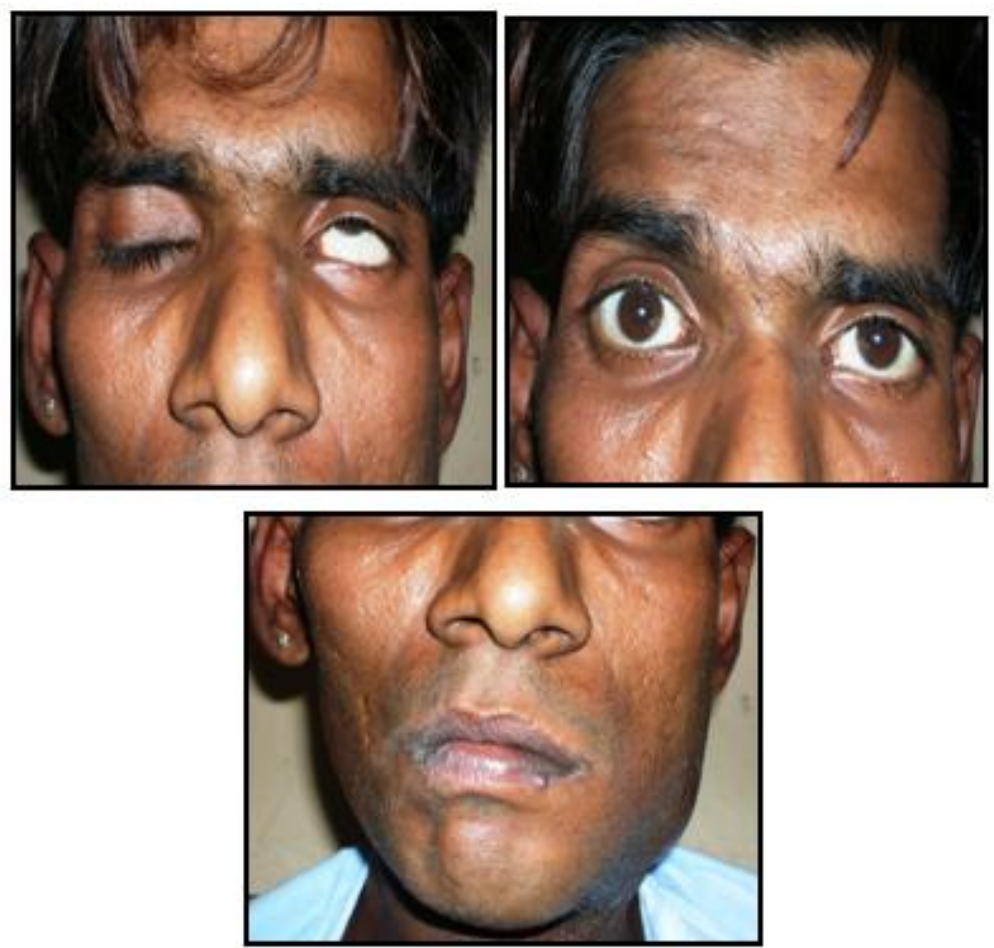

Figures showing signs of Bell's palsy where patient is unable to:

a) close eyes on affected side

b) produce wrinkles on forehead on affected side

c) blow mouth where air escapes from affected side

Provisional Diagnosis: Bell's palsy secondary to odontogenic

space infection

\section{Investigations and management:}

1) Inj. Tetanus toxoid $0.5 \mathrm{cc}$ was given intramuscularly on the day of examination.

2) The patients haemogram was done which showed predominant leukocytes. His HIV status was negative.

3) Orthopantomogram revealed infected, horizontally impacted mandibular

left third molar with unfavourable root configuration and infected mandibular left permanent second molar with unusually long roots.Also, mandibular right third molar was horizontally impacted and the mandibular right permanent second molar was infected with unusually long roots.

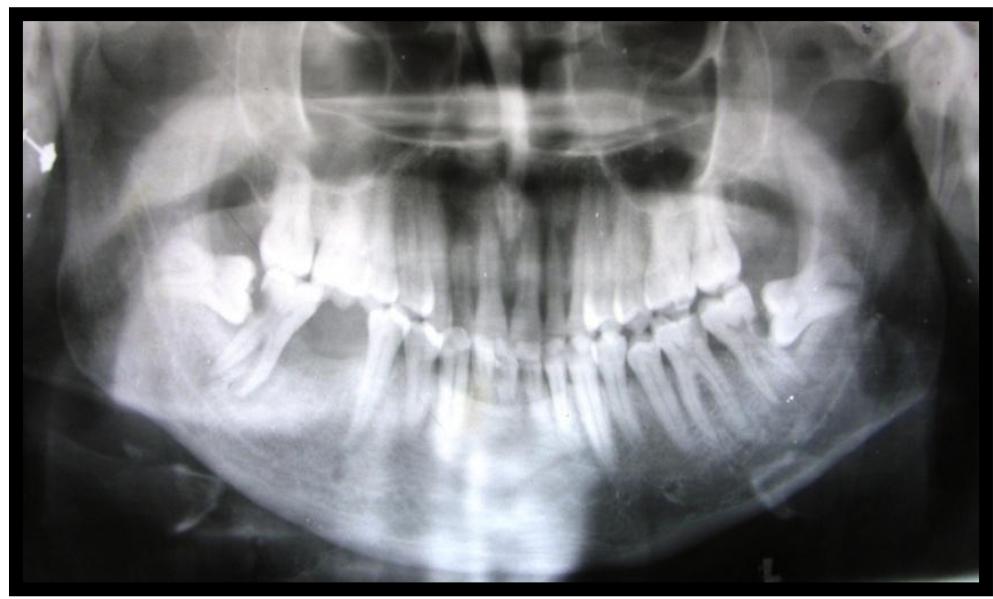




\section{Orthopantomogram Of Patient Reveals:}

a) Distal carious lesion with mandibular right \& left permanent second molar with periapical rarefaction.

b) Unusually long roots of the mandibular right and left permanent second molars.

c) Impacted mandibular right and left third molars.

4) Patients' chest radiograph was done as patient had past history of Koch's disease. The chest radiograph was reviewed by the physician who confirmed that there were no signs of reactivation or reinfection of Koch's disease.

4) CT Scan with contrast of the facial region was done. It showed diffuse spread of the infection in the masticator spaces on left side, left parotid region as well as down the left carotid sheath, displacing the vital structures.
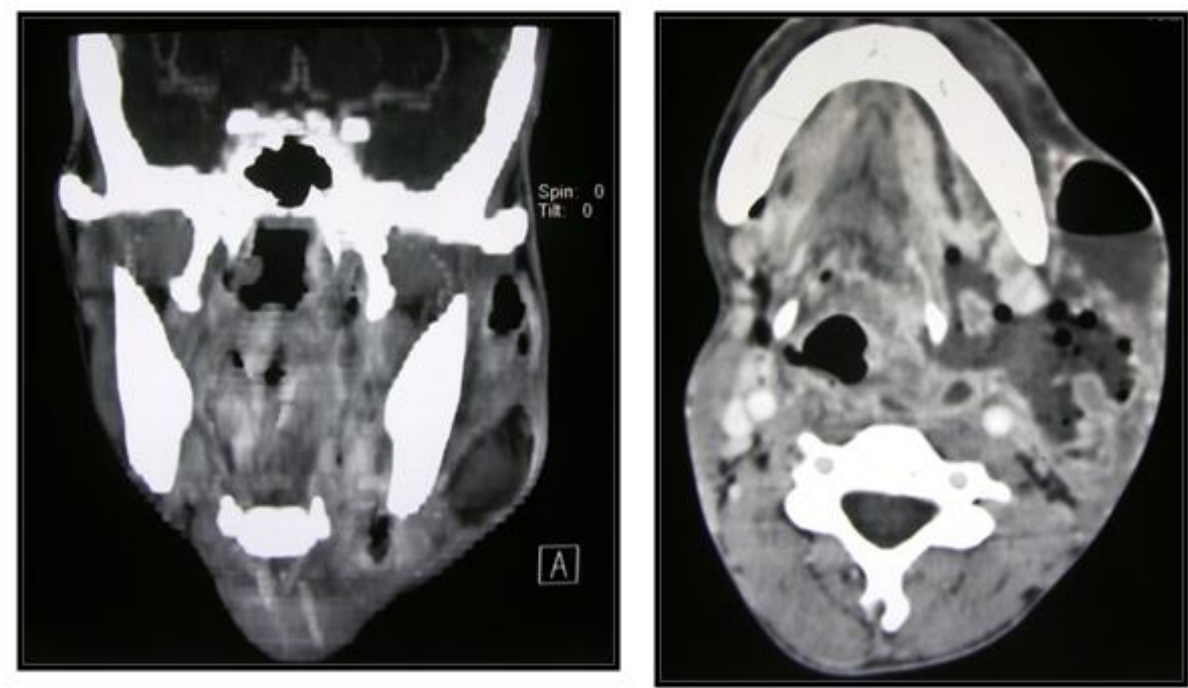

5) Neurology reference was done in view of the facial palsy to rule out any central cause for the nerve injury. However, no central cause for the nerve injury was identified.

6) Otorhinolaryngology reference was done to rule out any signs of nerve injury during it's course through the tympanic region. Next patient was given parenteral medication and intravenous fluids and once patients' general condition was stabilized, the abscess was drained extraorally. Copious amount of pus was drained and lot of necrotic material was expressed. The pus sample was sent for antibiotic culture and sensitivity testing. However, the reports were positive for gram positive streptococcal infection only.

2 days after the abscess was drained, patients' mouth opening Improved. The infected, horizontally impacted mandibular left third molar was removed surgically by sectioning of the tooth and the infected mandibular left permanent second molar was extracted. The infected mandibular left permanent second molar was found to have unusually long roots.
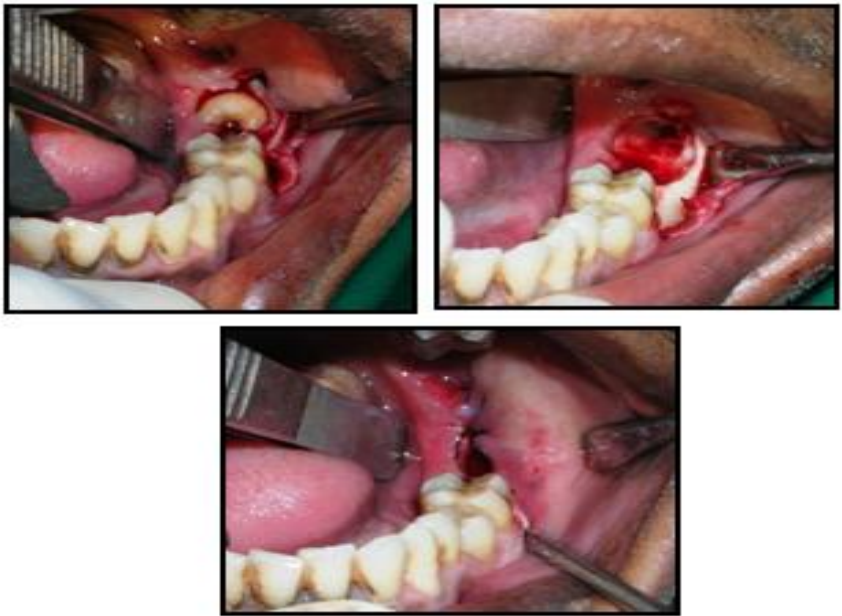
Figures showing the steps carried out during the surgical removal of the infected mandibular left second and third molars: Reflection of the mucoperiosteal flap, Debridement of the wound following removal of the focii of infection, Wound sutured loosely.

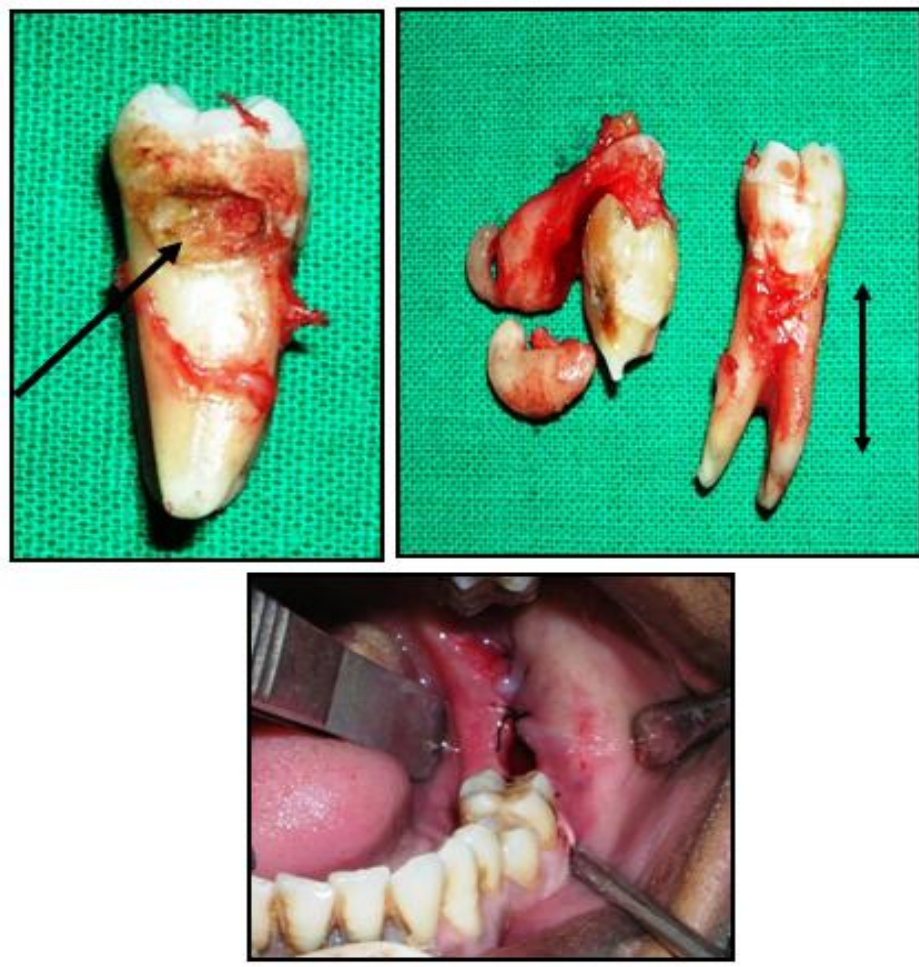

Figures showing the focii of infectionDistal carious defect on the mandibular left permanent second molar.

The unusually long roots of the mandibular left permanent second molar and the mandibular left third molar removed surgically by sectioning.

Once the pus discharge extraorally was negligible, patient was started with the steroid therapy. He was put on Tab. Methyl prednisolone 20mg three times a day for a period of a week and then gradually reduced to two times a day and finally once a day for a week. Along with the steroid therapy he was also started with active physiotherapy for rejuvenating the function of the facial nerve. Slowly it was found that he was able to achieve a good lip seal.

\section{Discussion}

The patient with acute facial palsy suffers not only the functional consequences of impaired facial motion, but also the psychological impact of a skewed facial appearance. A 1991 poll revealed that the level of discomfort Americans felt on meeting those with facial abnormalities was second only to that associated with interacting with the mentally ill, and it far exceeded anxiety about encountering the senile, mentally retarded, deaf, blind and those confined to a wheelchair. An acute facial palsy can have a dramatic effect on the quality of life, and disorders of the facial nerve pose a clinical challenge confronted by health professionals from a wide variety of disciplines. Although Sir Charles Bell resolved many questions about patterns of facial innervation, Bell's palsy, the disorder named for him, continues to generate substantial debate and study. Despite decades of expanded insights into Bell's and other acute facial palsies, no clear consensus has yet emerged on many aspects of their aetiology, evaluation and management. ${ }^{1}$

The facial nerve consists of a motor and a sensory part, the latter Known as the nervus intermedius. The motor root arises from a nucleus which lies deeply in the reticular formation of the lower part of the pons.

The sensory root arises from the genicular ganglion, which is situated on the geniculum of the facial nerve in the facial canal, behind the hiatus of the canal. The muscles supplied by the motor part of the facial nerve include 
muscles of the face, scalp, and auricle, the buccinator and platysma, the stapedius, the stylohyoideus, and posterior belly of the digastricus; it also contains some sympathetic motor fibers to the submaxillary and sublingual glands, and are conveyed through the chorda tympani nerve. The sensory part contains the fibers of taste for the anterior two-thirds of the tongue. ${ }^{2}$

\section{Branches of communication of the facial nerve:}

1) In the internal acoustic meatus, the facial nerve communicates with the acoustic nerve.

2) At the genicular ganglion, the facial nerve communicates with the sphenopalatine ganglion by the greater superficial petrosal nerve, with the otic ganglion by a branch which joins the lesser superficial petrosal nerve and with the sympathetic on the middle meningeal artery.

3) In the facial canal, the facial nerve communicates with the auricular branch of the vagus nerve.

4) At the exit from the stylomastoid foramen, the facial nerve communicates with the glossopharyngeal, vagus, great auricular and the auriculotemporal nerves

5) Behind the ear, the facial nerve communicates with the lesser occipital nerve.

6) On the face, the facial nerve communicates with the trigeminal nerve.

7) In the neck, the facial nerve communicates with the cutaneous cervical nerve.

\section{Branches of distribution of the facial nerve:}

1) Within the facial canal, facial nerve gives two branches, namely, nerve to stapedius muscle and chorda tympani.

2) At it's exit from the stylomastoid foramen, facial nerve gives three branches, namely, posterior auricular branch, digastrics branch and stylohyoid branch.

3) In the facial region, the facial nerve gives it's terminal branches. It divides into two main branches, namely temporofacial branch and cervicofacial branch. The temporofacial branch gives three branches, namely, the temporal, zygomatic and upper buccal branches. The cervicofacial branch gives another three branches, namely, the lower buccal, marginal mandibular and cervical branches.

Investigators have implicated few causes for development of Bell's palsy namely, viral infection, immunologic mechanisms and vascular aetiologies; the least common cause being odontogenic infection. Localization of a odontogenic infections is related to the anatomic position of the dental root from which it originated, especially in relationship to muscle attachments. Apart from haematogenous spread, odontogenic infections may spread through lymphatic vessels, or by direct continuity through loose connective tissues or by involvement of veins. In this case, the infected mandibular molars had unusually long roots which may have facilitated spread of infection to involve the submandibular space. Also, submandibular space has communications with the pterygomandibular space and the deep lobe of the parotid gland forms the posterior boundary of the pterygomandibular space. Pterygomandibular space infection is likely to affect the functioning of the terminal branches of the facial nerve, which may further be aggravated owing to ischaemic injury caused by long standing masticator space infections. ${ }^{3}$

A Computed Tomography scan with contrast is ideal for studying the facial nerve. The facial nerve cannot be identified on direct axial scans, but it's location can be approximated by the readily identifiable retromandibular vein. The facial nerve lies lateral to the retromandibular vein, and lateral displacement of the retromandibular vein is indicative of pathology arising in the deep lobe of parotid. Secondary infections of the parotid space may occur through contiguous spread from the adjacent parapharyngeal or masticator spaces, as well as through lymphatic spread.

Masticator space infections lead to trismus. Management of odontogenic infections needs a comprehensive assessment and management of the case. Incision and drainage of the abscess under suitable anaesthesia reduces the irritation of the masticator muscles thereby improving the interincisal opening. This further enables the maxillofacial surgeon to treat the odontogenic foci of infection. Careful assessment of the maxillary third molars is essential for successful management of infections associated with impacted mandibular third molars.Corticosteroids and physiotherapy may help in faster recovery of the nerve function.

\section{Conclusion}

The surgical skill in the management of odontogenic infections demands a careful understanding of the patients' general condition, comprehensive examination of the patient along with the radiological assessment and meticulous execution of the step-wise treatment plan with special emphasis on maintenance of patients airway. Also, the prognosis of cases of nerve injuries caused due to odontogenic infections is guarded. It depends, not only on successful management of the pathology, but also on the host response to the treatment, and this fact needs to be explained to the patients during initial consultations to prevent litigation. 
Finally, the management of pus in the neck continues to call for the surgeon's best judgement, his best skills, and often all his courage.

\section{References}

[1]. Neurotology. Robert E.Jackler, Derald E.Brackmann; Mosby publication.

[2]. Int. J. Morphol., 27(1) :183-186, 2009

[3]. Anatomy of the Facial Nerve and its Implication in the Surgical Procedures.

[4]. Antonio de Castro Rodrigues; Jesus Carlos Andreo; Laura de Freitas Menezes; Tatiana Pimentel Chinellato \& Geraldo Marco Rosa Júnior.

[5]. Int. J. Infectious Diseases; May 2009Volume 13, Issue 3, Pages 327-333.

[6]. Submandibular space infection: a potentially lethal infection.

[7]. Paolo Boscolo-Rizzo, Maria Cristina Da Mosto 